\title{
Normative argumentation and qualitative probability
}

\author{
Simon Parsons \\ Department of Electronic Engineering, Queen Mary and Westfield College, \\ Mile End Road, London E1 4NS, United Kingdom. \\ S.D.Parsons@qmw.ac.uk
}

\begin{abstract}
In recent years there has been a spate of papers describing systems for plausible reasoning which do not use numerical measures of uncertainty. Some of the most successful of these have been systems for argumentation, and there are advantages in considering the conditions under which such systems are normative. This paper discusses an extension to previous work on normative argumentation, exploring the properties of a particular normative approach to argumentation and suggesting some uses of it.
\end{abstract}

\section{Introduction}

In the last few years there have been a number of attempts to build systems for reasoning under uncertainty that are of a qualitative nature - that is they use qualitative rather than numerical values, dealing with concepts such as increases in belief and the relative magnitude of values. Between them, these systems address the problem of reasoning in situations in which knowledge is uncertain, but in which there is a limited amount of numerical information quantifying the degree of uncertainty. Three main classes of system can be distinguishedsystems of abstraction, infinitesimal systems, and systems of argumentation.

In systems of abstraction, $[5,14,19,22]$, the focus is often, though not always [3], on modelling how the probability of hypotheses changes when evidence is obtained and never commits to exact probability values. They thus provide an abstract version of probability theory which ignores the actual values of individual probabilities but which is nevertheless sufficient for planning and design [13] tasks. Infinitesimal systems $[10,23]$ deal with beliefs that are very nearly 1 or 0 , providing formalisms that handle order of magnitude probabilities. Infinitesimal systems may be used for diagnosis [4] as well as providing a general model of default reasoning. Systems of argumentation $[1,2,6,11,12,21]$ are based on the idea of constructing logical arguments for and against propositions, establishing the overall validity of such propositions by assessing the persuasiveness of the individual arguments.

However, unlike other qualitative systems, systems of argumentation do not always have a clear semantics. In particular, it is not always clear what it means to have an argument for or against something. As a result, in a previous paper 
[17] I made the suggestion that it might be beneficial to investigate whether systems of argumentation might be made normative in a probabilistic sense, thus combining the expressiveness of argumentation with the clear semantics of probability. This paper takes that work further, building a more expressive system, exploring its properties, providing soundness and completeness results and suggesting ways in which the system might be used.

\section{Introducing systems of argumentation}

In classical logic, an argument is a sequence of inferences leading to a conclusion. If the argument is correct, then the conclusion is true. In the system of argumentation proposed by Fox, Krause and colleagues [11] this traditional form of reasoning is extended to allow arguments to indicate support and doubt in propositions, as well as proving them. This is done by assigning two labels to every sentence that is deduced. One label, the grounds, identifies the argument for that sentence by indicating the formulae used in the deduction. The other label, the sign, indicates the force of the argument. The force can be taken to be the change in belief in the proposition warranted by the argument. This form of argumentation may be summarised by the following schema:

$$
\text { Database } \vdash_{A C R} \text { (Sentence, Grounds, Sign) }
$$

where $\vdash_{A C R}$ is a suitable consequence relation. This approach to argumentation is one of many, but it is the one which will be adopted in this paper. Having chosen to work with this form of argumentation, we then choose a particular formalisation of this form of argumentation to work with, creating a system which we will call $\mathcal{S} \mathcal{A}^{\prime \prime}$. Again there are many possibilities of which this formalisation is just one, particularly convenient, one. Others are explored in $[8,9,11,16]$. Since this formalisation is expressed in very general terms, $\mathcal{S} \mathcal{A}^{\prime \prime}$ can actually be refined to produce a number of different systems depending upon the precise interpretation one puts on the signs. Eventually a particular interpretation is selected, generating a system $\mathcal{N} \mathcal{A}^{\prime \prime}$, and the bulk of the paper investigates the properties and uses of this system.

We start the definition of $\mathcal{S} \mathcal{A}^{\prime \prime}$ with a set of atomic propositions $\mathcal{L}$. We also have a set of connectives $\{\rightarrow, \wedge, \neg\}$, and the following set of rules for building the well-formed formulae $(w f f \mathrm{~s})$ of the language.

- If $l \in \mathcal{L}$ then $l$ is a simple well-formed formula (swff).

- If $l$ is an swff, then $\neg l$ is an swff.

- If $l$ and $m$ are swffs then $l \wedge m$ is an swff.

- If $l$ and $m$ are swffs then $l \rightarrow m$ is an implicational well-formed formula (iwff).

- The set of all wffs is the union of the set of $s w f f \mathrm{~s}$ and the set of iwffs.

The reason for distinguishing the swffs and the iwffs is that whilst $\neg$ and $\wedge$ have their usual logical meaning, $\rightarrow$ does not represent material implication but 


$$
\begin{aligned}
& \mathrm{Ax} \frac{}{\Delta \vdash_{A C R}(S t,\{l\}, S g)}(l: S t: S g) \in \Delta \\
& \wedge-\mathrm{E} 1 \frac{\Delta \vdash_{A C R}\left(S t \wedge S t^{\prime}, G, S g\right)}{\Delta \vdash_{A C R}(S t, G, \text { conjelim }(S g))} \\
& \wedge-\mathrm{E} 2 \frac{\Delta \vdash_{A C R}\left(S t \wedge S t^{\prime}, G, S g\right)}{\Delta \vdash_{A C R}\left(S t^{\prime}, G, \text { conj }_{\mathrm{elim}}(S g)\right)} \\
& \wedge-\mathrm{I} \frac{\Delta \vdash_{A C R}(S t, G, S g) \quad \Delta \vdash_{A C R}\left(S t^{\prime}, G^{\prime}, S g^{\prime}\right)}{\Delta \vdash_{A C R}\left(S t \wedge S t^{\prime}, G \cup G^{\prime}, \operatorname{comb}_{\text {conj }}\left(S g, S g^{\prime}\right)\right)} \\
& \rightarrow-\mathrm{E} \frac{\Delta \vdash_{A C R}(S t, G, S g) \quad \Delta \vdash_{A C R}\left(S t \rightarrow S t^{\prime}, G^{\prime}, S g^{\prime}\right)}{\Delta \vdash_{A C R}\left(S t^{\prime}, G \cup G, \mathrm{comb}_{\mathrm{imp}}\left(S g, S g^{\prime}\right)\right)} \\
& \rightarrow-\mathrm{I} \frac{\Delta,(S t, \emptyset, S g) \vdash_{A C R}\left(S t^{\prime}, G, S g^{\prime}\right)}{\Delta \vdash_{A C R}\left(S t \rightarrow S t^{\prime}, G, \mathrm{Comb}_{\mathrm{imp}}^{-1}\left(S g, S g^{\prime}\right)\right)} \\
& \neg-\mathrm{E} 1 \frac{\Delta \vdash_{A C R}(\neg S t, G, S g)}{\Delta \vdash_{A C R}\left(S t, G, \operatorname{neg}_{\text {form }}(S g)\right)} \\
& \neg-\mathrm{I} \frac{\Delta \vdash_{A C R}(S t, G, S g)}{\Delta \vdash_{A C R}\left(\neg S t, G, \text { neg }_{\text {form }}(S g)\right)}
\end{aligned}
$$

Fig. 1. Argumentation Consequence Relation

a connection between the signs of antecedent and consequent. Thus there is a fundamental difference between the $s w f f s$ and the $i w f f \mathrm{~s}$ which will become clear later in the paper. The set of all wffs that may be defined using $\mathcal{L}$, may then be used to build up a database $\Delta$ where every item $d \in \Delta$ is a triple $(i: l: s)$ in which $i$ is a token uniquely identifying the database item (for convenience we will use the letter ' $i$ ' as an anonymous identifier), $l$ is a $w f f$, and $s$ is a statement about belief in $l$. With this formal system, we can take a database and use the argumentation consequence relation $\vdash_{A C R}$ defined in Figure 1 to build arguments for propositions that we are interested in $^{1}$.

Typically we have several arguments for a given proposition, and so flatten them to get a single sign. Thus we have a function Flat $(\cdot)$ from a set of arguments A for a proposition $l$ from a particular database $\Delta$ to the pair of that proposition and some overall measure of validity:

$$
\operatorname{Flat}(\mathbf{A})=\langle l, v\rangle
$$

where $\mathbf{A}=\left\{(l, G, S g) \mid \Delta \vdash_{A C R}(l, G, S g)\right\}$, and $v$ is the result of a suitable combination of the $S g$ that takes into account the structure of the the arguments.

\footnotetext{
${ }^{1}$ The change from the triple (Identifier, Sentence, Sign) in the database to the argument (Sentence, Set of Identifiers, Sign) is to make a distinction between database items and derived sentences.
} 
The value of $v$ is calculated by another function flat:

$$
v=\operatorname{flat}\left(\left\{\left\langle G_{i}, S g_{i}\right\rangle \mid\left(l, G_{i}, S g_{i}\right) \in \mathbf{A}\right\}\right)
$$

Together $\mathcal{L}$, the rules for building the formulae, the connectives, and $\vdash_{A C R}$ define $\mathcal{S} \mathcal{A}^{\prime \prime}$. In fact, $\mathcal{S} \mathcal{A}^{\prime \prime}$ is really the basis of a family of systems of argumentation, because one can define a number of variants of $\mathcal{S} \mathcal{A}^{\prime \prime}$ by using different sets of signs. Each set will have its own set of functions conjelim, comb $b_{\text {conj }}, \operatorname{comb}_{\text {imp }}, \operatorname{comb}_{\text {imp }}^{-1}$

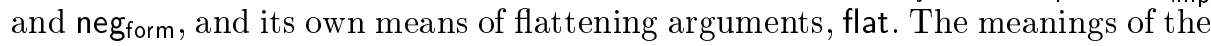
signs, flattening functions, and combination functions delineate the semantics of the system of argumentation. The purpose of this paper is to suggest a way in which these functions may be given a specific probabilistic interpretation. The reason for doing this is to provide a system of argumentation, $\mathcal{N} \mathcal{A}^{\prime \prime}$, which is normative in the sense that it accords to the norms of probability theory.

\section{A probabilistic semantics}

The idea behind the semantics is to provide a precise characterisation of the intuitive idea that constructing an argument which supports a proposition is a reason for one to increase one's belief in that proposition and that constructing an argument against a proposition is a reason for decreasing one's belief in that proposition. Taking the position that a degree of belief may be expressed as a probability it is possible to modify the notion of a probabilistic influence in qualitative probabilistic networks (QPNs) [22] to give the signs of $\mathcal{N} \mathcal{A}^{\prime \prime}$ a probabilistic interpretation.

\subsection{The meaning of formulae}

In particular we take triples $(i: l: \uparrow)$ to denote the fact that $\operatorname{Pr}(l)$ increases, and similar triples $(i: l: \downarrow)$, to denote the fact that $\operatorname{Pr}(l)$ decreases. Triples $(i: l: \leftrightarrow)$, denote the fact that $\operatorname{Pr}(l)$ is known to neither increase nor decrease. It should be noted that the triple $(i: l: \uparrow)$ indicates that the change in value of $\operatorname{Pr}(l)$ either goes up, or does not change - this inclusive interpretation of the notion of "increase" is taken from QPNs - and of course a similar proviso applies to $(i: l: \downarrow)$. Since we want to reason about changes in belief which equate to the usual logical notion of proof, we also consider changes in belief to 1 and decrease in belief to 0 , indicating these by the use of the symbols $\Uparrow$ and $\Downarrow$. The meaning of a proposition $(i: l: \Uparrow)$ is that the probability of $l$ becomes 1 , while $(i: l: \Downarrow)$ means that the probability of $l$ becomes 0 . We also have triples $(i: l: \uparrow)$ which indicate that the change in $\operatorname{Pr}(l)$ is unknown.

Implications, by which we mean iwffs, can be given a probabilistic interpretation by making the triple $(i: a \rightarrow c:+)$ denote the fact that:

$$
\operatorname{Pr}(c \mid a, X) \geq \operatorname{Pr}(c \mid \neg a, X)
$$


for all $X \in\{x, \neg x\}$ for which there is a triple $(i: x \rightarrow c: s)$ (where $s$ is any sign) or $(i: \neg x \rightarrow c: s)$, while the triple $(i: a \rightarrow c:-)$ denotes the fact that:

$$
\operatorname{Pr}(c \mid a, X) \leq \operatorname{Pr}(c \mid \neg a, X)
$$

again for all $X \in\{x, \neg x\}$ for which there is a triple $(i: x \rightarrow c: s)$ or $(i: \neg x \rightarrow$ $c: s)$. As a result an implication $(i: a \rightarrow c:+)$ means that there is a probability distribution over the formulae $c$ and $a$ such that an increase in the probability of $a$ makes $c$ more likely to be true, and an implication $(i: a \rightarrow c:-)$ means that there is a probability distribution over the propositions $c$ and $a$ such that an increase in the probability of $a$ makes $c$ less likely to be true. We do not make much use of triples such as $(i: c \rightarrow a: 0)$ since they have no useful effect but include them for completeness- $(i: c \rightarrow a: 0)$ indicates that $\operatorname{Pr}(c)$ does not change when $\operatorname{Pr}(a)$ changes. We also have implications such as $(i: a \rightarrow c: ?)$ which denotes the fact that the relationship between $\operatorname{Pr}(c \mid a, X)$ and $\operatorname{Pr}(c \mid \neg a, X)$ is not known, so that if the probability of $a$ increases it is not possible to say how the probability of $c$ will change. With this interpretation, implications between atomic propositions correspond to qualitative influences in QPNs. As a result of this link to QPNs we require that implications are causally directed, by which we mean that the antecedent is a cause of the consequent. This is the usual restriction imposed in probabilistic networks [20].

It should be noted that the effect of declaring that there is an implication $(i: a \rightarrow c:+)$ is to create considerable constraints on the probability distribution over $a$ and $c$. In fact we have:

Theorem 1. A consequence of the probabilistic semantics is that an implication $(i: a \rightarrow c:+)$ places the same restrictions over the probabilities of $a$ and $c$ as $(i: a \rightarrow \neg c:-),(i: \neg a \rightarrow c:-),(i: \neg a \rightarrow \neg c:+)$ and $(i: c \rightarrow a:+)$, $(i: c \rightarrow \neg a:-),(i: \neg c \rightarrow a:+),(i: \neg c \rightarrow \neg a:+)$.

Proof: The condition $\operatorname{Pr}(c \mid a, X) \geq \operatorname{Pr}(c \mid \neg a, X)$ is exactly that for $(i: \neg a \rightarrow$ $c:-)$, and implies $1-\operatorname{Pr}(c \mid a, X) \leq 1-\operatorname{Pr}(c \mid \neg a, X)$ so that $\operatorname{Pr}(\neg c \mid a, X) \leq$ $\operatorname{Pr}(\neg c \mid \neg a, X)$. This is the condition for $(i: a \rightarrow \neg c:-)$ and $(i: \neg a \rightarrow \neg c:+)$. This takes care of the restrictions on reasoning from $a$ to $c$. For the reverse cases we recall that $\operatorname{Pr}(c \mid a, x) \geq \operatorname{Pr}(c \mid \neg a, x)$ implies $\operatorname{Pr}(a \mid c, y) \geq \operatorname{Pr}(a \mid \neg c, y)$ [22].

Clearly analogous restrictions are imposed by implications like $(i: a \rightarrow c:-)$. We also have categorical implications which allow propositions to be proved true or false. In particular, an implication $(i: a \rightarrow c:++)$ indicates that when $a$ is known to be true, then so is $c$. Thus it denotes a constraint on the probability distribution across $a$ and $c$ such that if $\operatorname{Pr}(a)$ becomes 1 , so does $\operatorname{Pr}(c)$. This requires that:

$$
\operatorname{Pr}(c \mid a, X)=1
$$

for all $X \in\{x, \neg x\}$ for which there is a triple $(i: x \rightarrow c: s)$ or $(i: \neg x \rightarrow$ $c: s)$ [15]. Note that this type of implication also conforms to the conditions for implications labelled with + , and that if $\operatorname{Pr}(c \mid \neg a, x)=1$ then $\operatorname{Pr}(c)$ is 
always equal to $\operatorname{Pr}(a)$. Similarly, a probabilistic interpretation of an implication $(i: a \rightarrow c:--)$ which denotes the fact that if $a$ is true, $c$ is false requires that:

$$
\operatorname{Pr}(c \mid a, X)=0
$$

for all $X \in\{x, \neg x\}$ for which there is a triple $(i: x \rightarrow c: s)$ or $(i: \neg x \rightarrow c: s)$. There are two more types of categorical implication which are symmetric to those already introduced. The first is of the form $(i: a \rightarrow c:-+)$ which denotes the constraint:

$$
\operatorname{Pr}(c \mid \neg a, X)=1
$$

for all $X \in\{x, \neg x\}$ for which there is a triple $(i: x \rightarrow c: s)$ or $(i: \neg x \rightarrow c: s)$. The second is of the form $(i: a \rightarrow c:+-)$ which denotes the constraint:

$$
\operatorname{Pr}(c \mid \neg a, X)=0
$$

for all $X \in\{x, \neg x\}$ for which there is a triple $(i: x \rightarrow c: s)$ or $(i: \neg x \rightarrow c: s)$. Unsurprisingly, the introduction of categorical implications imposes restrictions on other implications involving the same formulae.

Theorem 2. A consequence of the probabilistic semantics is that an implication $(i: a \rightarrow c:++)$ places the same restrictions over the probabilities of $a$ and $c$ as $(i: a \rightarrow \neg c:--),(i: \neg a \rightarrow c:-+)$ and $(i: \neg a \rightarrow \neg c:+-)$, and implies that for all other implications $(i: x \rightarrow c: s)$ where $x \neq a$, it is the case that $s \in\{++,+,-,-+\}$.

Proof: The implication $(i: a \rightarrow c:++)$ requires $\operatorname{Pr}(c \mid a, X)=1$ which is exactly the constraint imposed by $(i: a \rightarrow \neg c:-+) \cdot \operatorname{Pr}(c \mid a, X)=1$ implies $\operatorname{Pr}(\neg c \mid a, X)=0$ which is the condition imposed by $(i: a \rightarrow \neg c:--)$ and $(i: \neg a \rightarrow \neg c:+-)$. The restriction on implications such as $(i: x \rightarrow c: s)$ follows directly from the mutual incompatibility of the constraints on the conditional probabilities imposed by the categorical implications [15].

This property is exactly what we should expect. If $\operatorname{Pr}(a)$ is related to $\operatorname{Pr}(c)$ then it is also related to $\operatorname{Pr}(\neg c)$, and its negation is related to both $\operatorname{Pr}(c)$ and $\operatorname{Pr}(\neg c)$. Furthermore, if the occurrence of $a$ proves $c$ to be true, then no other evidence can change this conclusion so implications labelled +- or -- may not have $c$ as their consequent. Similar results hold for other types of categorical implication.

Theorem 3. A consequence of the probabilistic semantics is that an implication $(i: a \rightarrow c:++)$ or $(i: a \rightarrow c:-+)$ places the same restrictions over the probabilities of $a$ and $c$ as $(i: c \rightarrow a:+),(i: c \rightarrow \neg a:-),(i: \neg c \rightarrow a:-)$ and $(i: \neg c \rightarrow \neg a:+)$.

Proof: An categorical implication $(i: a \rightarrow c:++)$ or $(i: a \rightarrow c:-+)$ is just a more extreme version of $(i: a \rightarrow c:+)$, and while it won't necessarily reverse to give a categorical implication, it will reverse just like $(i: a \rightarrow c:+)$ so the result follows from Theorem 1 .

Again, analogous results hold for the other kinds of categorical implications. 


\begin{tabular}{|l|llllll|}
\hline & $\Uparrow$ & $\uparrow$ & $\leftrightarrow$ & $\downarrow$ & $\Downarrow$ & $\uparrow$ \\
\hline$\Uparrow$ & $\Uparrow$ & $\uparrow$ & $\uparrow$ & $\uparrow$ & $\uparrow$ & $\uparrow$ \\
$\uparrow$ & $\uparrow$ & $\uparrow$ & $\uparrow$ & $\uparrow$ & $\uparrow$ & $\uparrow$ \\
$\leftrightarrow$ & $\uparrow$ & $\uparrow$ & $\leftrightarrow$ & $\downarrow$ & $\downarrow$ & $\uparrow$ \\
$\downarrow$ & $\uparrow$ & $\uparrow$ & $\downarrow$ & $\downarrow$ & $\downarrow$ & $\uparrow$ \\
$\downarrow$ & $\uparrow$ & $\uparrow$ & $\downarrow$ & $\downarrow$ & $\Downarrow$ & $\uparrow$ \\
$\uparrow$ & $\uparrow$ & $\uparrow$ & $\uparrow$ & $\uparrow$ & $\uparrow$ & $\uparrow$ \\
\hline
\end{tabular}

(a)

\begin{tabular}{|c|cccccccc|}
\hline & ++ & +- & + & 0 & - & -+ & -- & $?$ \\
\hline$\Uparrow$ & $\Uparrow$ & $\uparrow$ & $\uparrow$ & $\leftrightarrow$ & $\downarrow$ & $\Downarrow$ & $\downarrow$ & $\downarrow$ \\
$\uparrow$ & $\uparrow$ & $\uparrow$ & $\uparrow$ & $\leftrightarrow$ & $\downarrow$ & $\downarrow$ & $\downarrow$ & $\uparrow$ \\
$\leftrightarrow$ & $\leftrightarrow$ & $\leftrightarrow$ & $\leftrightarrow$ & $\leftrightarrow$ & $\leftrightarrow$ & $\leftrightarrow$ & $\leftrightarrow$ & $\leftrightarrow$ \\
$\downarrow$ & $\downarrow$ & $\downarrow$ & $\downarrow$ & $\leftrightarrow$ & $\uparrow$ & $\uparrow$ & $\uparrow$ & $\uparrow$ \\
$\Downarrow$ & $\downarrow$ & $\Downarrow$ & $\downarrow$ & $\leftrightarrow$ & $\uparrow$ & $\uparrow$ & $\Uparrow$ & $\uparrow$ \\
$\uparrow$ & $\uparrow$ & $\uparrow$ & $\uparrow$ & $\leftrightarrow$ & $\uparrow$ & $\uparrow$ & $\uparrow$ & $\uparrow$ \\
\hline
\end{tabular}

(b)

Table 1. Conjunction introduction comb $b_{\text {conj }}(a)$ and implication elimination combimp (b)

\subsection{The proof rules}

We now turn to providing a probabilistic interpretation for the functions used to build arguments starting with conjunction introduction and elimination. When introducing conjunction we have:

Definition 4. The function $\mathrm{comb}_{\text {conj }}: S g \in\{\Uparrow, \uparrow, \leftrightarrow, \downarrow, \Downarrow, \uparrow\} \times S g^{\prime} \in\{\Uparrow, \uparrow$, $\leftrightarrow, \downarrow, \Downarrow, \uparrow\} \mapsto S g^{\prime \prime} \in\{\Uparrow, \uparrow, \leftrightarrow, \downarrow, \downarrow, \uparrow\}$ is specified by Table 1(a) where, as with all combinator tables in this paper, the first argument is taken from the first column and the second argument is taken from the first row.

When eliminating a conjunction with sign $S g$ we assign both conjuncts the sign conjelim $(S g)$.

Definition 5. The function conjelim : $S g \in\{\Uparrow, \uparrow, \leftrightarrow, \downarrow, \Downarrow, \uparrow\} \mapsto S g^{\prime} \in\{\Uparrow, \uparrow, \leftrightarrow$, $\downarrow, \Downarrow, \uparrow\}$ is as follows:

$$
\operatorname{conj}_{\text {elim }}(S g)= \begin{cases}\Uparrow & \text { if } \mathrm{Sg}=\Uparrow \\ \downarrow & \text { otherwise }\end{cases}
$$

What this means is that most of the time it is not possible to determine how the probability of the individual conjuncts change. This is an unfortunate but unavoidable property of probability theory.

To deal with implication we need two further functions, comb imp $_{\text {p }}$ to establish the sign of a formula generated by the rule of inference $\rightarrow-\mathrm{E}$, and $\mathrm{comb}_{\mathrm{imp}}^{-1}$ to establish the sign of an implication generated by $\rightarrow$-I. This means that the main use of comb $b_{\text {imp }}$ is to combine the change in probability of a formula $a$, say, with the constraint that the probability of $a$ imposes upon the probability of another formula $c$. Since this constraint is expressed in exactly the same way as qualitative influences are in QPNs, comb $b_{\text {imp }}$ performs the same function as $\otimes$ [22], and is merely an extension of it.

Definition 6. The function comb $\mathrm{bimp}_{\mathrm{p}}: S g \in\{\Uparrow, \uparrow, \leftrightarrow, \downarrow, \downarrow, \uparrow\} \times S g^{\prime} \in\{++,+-$, $+, 0,-,-+,--, ?\} \mapsto S g^{\prime \prime} \in\{\Uparrow, \uparrow, \leftrightarrow, \downarrow, \Downarrow, \uparrow\}$ is specified by Table 1(b).

Note the asymmetry in the table which stems from the definition of the categorical implications. If the asymmetry did not exist, categorical implications would 
be close to logical bi-implication. As one might guess from its name, the function $\mathrm{comb}_{\mathrm{imp}}^{-1}$ is merely the inverse of comb $\mathrm{imp}_{\mathrm{m}}$ :

Definition 7. The function $\mathrm{comb}_{\mathrm{imp}}{ }^{-1}: S g \in\{\Uparrow, \uparrow, \leftrightarrow, \downarrow, \Downarrow, \uparrow\} \times S g^{\prime} \in\{\Uparrow, \uparrow, \leftrightarrow$, $\downarrow, \Downarrow, \uparrow\} \mapsto S g^{\prime \prime} \in\{++,+-,+, 0,-,-+,--, ?\}$ is specified by Table 2(a). Blank spaces represent impossible combinations.

The rules for handling negation are applicable only to $s w f f s$ and permit the negation to be either introduced or eliminated by reversing the direction of change of the sign, for example allowing $(i: \neg a: \uparrow)$ to be rewritten as $(i: a: \downarrow)$. This leads to the definition of negform:

Definition 8. The function neg form : $(i: \neg a: s), s \in\{\Uparrow, \uparrow, \leftrightarrow, \downarrow, \downarrow, \uparrow\} \mapsto(i: a:$ $\left.s^{\prime}\right), s^{\prime} \in\{\Uparrow, \uparrow, \leftrightarrow, \downarrow, \Downarrow, \uparrow\}$ relates $s$ to $s^{\prime}$ by Table $2(\mathrm{~b})$.

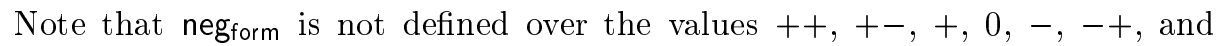
-- . Although an implication $(i: a \rightarrow b:+)$ has a kind of inverse relation with $(i: a \rightarrow b:-)$, there is no such relation with $(i: \neg(a \rightarrow b): s)$-indeed, $(i: \neg(a \rightarrow b): s)$ is not even an implication, since its main connective is $\neg$-and because of this it is not possible to apply neg form to an implication.

Having defined the combination of formulae to build arguments, we need to specify suitable function flat to be used to assess the overall strength of several arguments for the same formula. Now, in general, the overall strength of several arguments will be influenced by the interaction between steps in the arguments, and it will be necessary to take into account the grounds of each argument when flattening them in order to correctly handle any dependencies between the values of the formulae concerned. This is why flat is defined over the grounds as well as the signs of the arguments it operates on. However, in $\mathcal{N} \mathcal{A}^{\prime \prime}$, because the effect of each implication is defined to occur whatever other arguments are formed (this is a result of the constraint imposed on the conditional probabilities by the implications), all combinations are completely local, and the structure of the arguments may be disregarded when flattening (for exactly the same reason as it is in QPNs [5]). As a result, flat is simply the iterated application of the function

\begin{tabular}{|c|c|c|c|}
\hline & $\Uparrow \uparrow \leftarrow$ & $\leftrightarrow \quad \downarrow \quad \Downarrow$ & \\
\hline$\Uparrow$ & +++0 & $0---$ & $?$ \\
\hline$\uparrow$ & +0 & - & ? \\
\hline$\leftrightarrow$ & 0 & & \\
\hline$\downarrow$ & -0 & $0+$ & ? \\
\hline$\Downarrow$ & -+-0 & $0++-$ & t. \\
\hline$\uparrow$ & 0 & & \\
\hline
\end{tabular}

(a)

$$
\begin{array}{l|lll}
s & \Uparrow \leftrightarrow \downarrow \Downarrow \downarrow \\
\hline s^{\prime} \Downarrow \downarrow \leftrightarrow \uparrow \uparrow \uparrow
\end{array}
$$

(b)

\begin{tabular}{|c|cccccc}
\hline & $\Uparrow$ & $\uparrow$ & $\leftrightarrow$ & $\downarrow$ & $\Downarrow$ & $\uparrow$ \\
\hline$\Uparrow$ & $\Uparrow$ & $\Uparrow$ & $\Uparrow$ & $\Uparrow$ & & $\uparrow$ \\
$\uparrow$ & $\Uparrow$ & $\uparrow$ & $\uparrow$ & $\uparrow$ & $\Downarrow$ & $\uparrow$ \\
$\leftrightarrow$ & $\Uparrow$ & $\uparrow$ & $\leftrightarrow$ & $\downarrow$ & $\Downarrow$ & $\uparrow$ \\
$\downarrow$ & $\Uparrow$ & $\uparrow$ & $\downarrow$ & $\downarrow$ & $\Downarrow$ & $\uparrow$ \\
$\Downarrow$ & & $\Downarrow$ & $\Downarrow$ & $\Downarrow$ & $\Downarrow$ & $\Downarrow$ \\
$\uparrow$ & $\Uparrow$ & $\uparrow$ & $\uparrow$ & $\uparrow$ & $\Downarrow$ & $\uparrow$ \\
\hline
\end{tabular}

(c)

Table 2. Implication introduction $\mathrm{comb}_{\mathrm{imp}}^{-1}(\mathrm{a})$, negation of $\operatorname{swff} \mathrm{s}$ neg $\mathrm{form}_{\mathrm{m}}(\mathrm{b})$, and flattening flat $(c)$. 
$\oplus$ - an extended version of the qualitative addition function used by QPNs:

$$
\text { flat }\left(\left\{\left\langle G_{i}, S g_{i}\right\rangle\right\}\right)=\bigoplus_{i} S g_{i}
$$

where the correct way to combine the changes in probability using $\oplus$ is as follows:

Definition 9. The function $\oplus: S g \in\{\Uparrow, \uparrow, \leftrightarrow, \downarrow, \downarrow, \uparrow\} \times S g^{\prime} \in\{\Uparrow, \uparrow, \leftrightarrow, \downarrow, \Downarrow$, $\uparrow\} \mapsto S g^{\prime \prime} \in\{\Uparrow, \uparrow, \leftrightarrow, \downarrow, \downarrow, \uparrow\}$ is specified by Table 2 (c). Blank spaces represent impossible combinations.

\section{Example}

For an illustration of some of the reasoning possible in $\mathcal{N} \mathcal{A}^{\prime \prime}$, consider the following database. This encodes the information that three events have a bearing on whether or not I lose my job-being ill makes it more likely I will lose my job, doing good research makes it less likely I will lose my job, and embezzling money makes it certain I will lose my job-while being ill makes it more likely I will go to hospital:

$$
\begin{aligned}
& r 1: \text { good_research } \rightarrow \text { lose_job }:-\quad \Delta_{1} \\
& r 2: \text { ill } \rightarrow \text { lose_job }:+ \\
& r 3: \text { embezzle_money } \rightarrow \text { lose_job }:++ \\
& r 4: \text { ill } \rightarrow \text { hospital }:+ \\
& f 1: \text { good_research }: \Uparrow \\
& f 2: i l l: \Uparrow
\end{aligned}
$$

It also becomes known that I am ill and do good research (this is a fictional example). From this information we can build the arguments:

$$
\begin{aligned}
& \left.\Delta_{1} \vdash_{A C R} \text { (hospital, }\{f 2, r 4\}, \uparrow\right) \\
& \Delta_{1} \vdash_{A C R}\left(\text { lose }_{-} j o b,\{f 1, r 1\}, \downarrow\right) \\
& \Delta_{1} \vdash_{A C R}\left(\text { lose }_{-} j o b,\{f 2, r 2\}, \uparrow\right)
\end{aligned}
$$

The first argument means that I am increasingly likely to go to hospital. The second two arguments will flatten to give $\uparrow$, indicating that it is impossible to say how the probability of losing my job will change. These are exactly the conclusions that would be drawn by the equivalent QPN illustrating the fact that $\mathcal{N} \mathcal{A}^{\prime \prime}$ is capable of representing binary QPNs (as discussed in [16]) and can be regarded as a mechanism for the construction of binary QPNs. If we now add the fact that I am known to embezzle money from my employer:

$$
\text { ( } f 4: \text { : embezzle_money }: \Uparrow)
$$

to the database, we can build a new argument:

$$
\Delta_{3} \vdash_{A C R}\left(l_{0 s e} j o b,\{f 4, r 3\}, \Uparrow\right)
$$

meaning that the arguments about my loss of job will now flatten to give $\Uparrow$, so that my loss of job is assured. Thus $\mathcal{N A}^{\prime \prime}$ is capable of a form of defeasible reasoning in which certain information can outweigh previously known uncertain information. 


\section{$5 \quad$ Soundness and completeness}

Armed with the interpretation introduced in Section $3, \mathcal{N} \mathcal{A}^{\prime \prime}$ has a probabilistic semantics. However, the results presented so far have a rather baroque appearance, and so might seem ad hoc to the sceptical reader. However, they are not. The proof mechanism given above is provably sound for the propagation of changes in probability, as shown by the following result:

Theorem 10. The construction and flattening of arguments in $\mathcal{N} \mathcal{A}^{\prime \prime}$ is sound with respect to probability theory.

Proof: The soundness of $\mathcal{N} \mathcal{A}^{\prime \prime}$ follows immediately from the soundness of the the way in which changes in probabilities are propagated and flattened and thus from the soundness of the proof rules and combination tables.

(Conjunction introduction): Consider the probabilities $\operatorname{Pr}(a)$ and $\operatorname{Pr}(b)$ of the two propositions being conjoined. $\operatorname{Pr}(a \wedge b)=\operatorname{Pr}(a) \cdot \operatorname{Pr}(b \mid a)=\operatorname{Pr}(a \mid$ $b) . \operatorname{Pr}(b)$. Thus if at least one of $\operatorname{Pr}(a)$ and $\operatorname{Pr}(b)$ increases and the other does not decrease, then $\operatorname{Pr}(a \wedge b)$ will increase. If one increases and one decreases, then the change in $\operatorname{Pr}(a \wedge b)$ cannot be determined. If both increase to $1, \operatorname{Pr}(a \wedge b)$ increases to 1. Similar reasoning completes the proof.

(Conjunction elimination): There are two parts to the proof. One for the part of the function that gives $\Uparrow$ and one for the part that gives $\uparrow$. For the first, the following suffices - the only way in which $\operatorname{Pr}(a \wedge b)$ can increase to 1 is if both $\operatorname{Pr}(a)$ and $\operatorname{Pr}(b)$ increase to 1 (since the notion of 'increasing to 1' takes into account the fact values may have been 1 all along). For the second part we need the following argument. Giving any sign as $\uparrow$ is always sound (since it means that nothing at all is being said about the relevant probability). However, it is also possible to prove that no more precise rule can be proposed. This is done by considering what the probability of $\operatorname{Pr}(a \wedge b)$ should be if $\operatorname{Pr}(a)=\uparrow$ and $\operatorname{Pr}(b)=\downarrow$. The answer is that $\operatorname{Pr}(a \wedge b)$ can either increase, decrease, or not change depending on the relative magnitudes of the changes in $\operatorname{Pr}(a)$ and $\operatorname{Pr}(b)$. Turning this around, it is clear that no firm conclusions about changes in $\operatorname{Pr}(a)$ and $\operatorname{Pr}(b)$ can be drawn from particular changes in $\operatorname{Pr}(a \wedge b)$ other than $\operatorname{Pr}(a \wedge b)=\Uparrow$.

(Implication elimination): First consider implications labelled with + . From the definition of such implications it is clear that combining any increase in probability with an implication labelled + will generate a possible increase in probability, in other words $\uparrow$. Similarly combining any decrease in probability with an implication labelled + will generate $\downarrow$, combining no change in probability with such an implication will generate $\leftrightarrow$, and combining a change of $\uparrow$ with such an implication will generate $\uparrow$. An implication labelled - will also give $\leftrightarrow$ when combined with no change in probability and $\uparrow$ when combined with $\uparrow$, but otherwise will have the opposite behaviour to that of an implication labelled + . From its definition it is clear that an implication labelled ++ will behave like an implication labelled + except when combined with a change $\Uparrow$ when it will generate a change of $\Uparrow$. The results for other implications can be obtained analogously. 
(Implication introduction): The soundness of Table 2(a) follows directly from that of Table 1 (b). Where only one possible type of implication can give $S g^{\prime}$ the relevant sign is given by the table, where two or more can give $S g^{\prime}$, for instance when $S g=\uparrow$ and $S g^{\prime}=\uparrow$ when the sign of the implication could be,++-+ or + , the most inclusive sign is given, + in the case of our example since it subsumes ++ and -+ .

(Negation elimination and introduction): Consider a proposition $a$. If $\operatorname{Pr}(a)$ increases to 1 then clearly $\operatorname{Pr}(\neg a)$ decreases to zero, and if $\operatorname{Pr}(\neg 1)$ increases to 1 then clearly $\operatorname{Pr}(a)$ decreases to zero. This takes care of the function for $\Downarrow$ and $\Uparrow$. The other cases are handled similarly.

(Flattening): Table 2(c) follows directly from qualitative addition [22] and the fact that categorical changes in probability cannot be altered by non-categorical changes - the latter follows from the definition of categorical implications [15]. The spaces in the table follow from Property 2.

The other thing that might worry the sceptical reader is the completeness of $\mathcal{N} \mathcal{A}^{\prime \prime}$. However, since we only allow the initial database to contain implications $\left(i: S t \rightarrow S t^{\prime}: S g\right)$ where $S t$ is a direct cause of $S t^{\prime}$ we have the following result:

Theorem 11. The construction and flattening of arguments in $\mathcal{N} \mathcal{A}^{\prime \prime}$ is complete with respect to probability theory for reasoning in a causal direction.

Proof: Immediate from the definition of $\vdash_{A C R}$ and the causal direction of the implications - all possible causally directed inferences can be made by the application of the appropriate proof rules.

Causal completeness has its limitations, since from a database consisting of an implication $(r 1: a \rightarrow b:+)$ and a fact $(f 1: b: \uparrow)$ no arguments may be built using $\vdash_{A C R}$, yet using probability theory one can infer that $\operatorname{Pr}(a)$ increases. Extending $\mathcal{N} \mathcal{A}^{\prime \prime}$ to cope with this kind of evidential reasoning is straightforward and is discussed in [18].

\section{Discussion}

The probabilistic semantics of $\mathcal{N} \mathcal{A}^{\prime \prime}$ gives us two things. Firstly, it gives us a precise probabilistic notion of what it means to have an argument for something. If we accept the probabilistic interpretation of $w f f s$ then given a database of causal relations (implications) and evidence which leads to some changes in belief we can infer what changes in belief are implied. Only those propositions supported by sound arguments will undergo a change in belief and only those propositions for which an argument may be built have a change of belief warranted in them. Secondly, the semantics gives us a means of determining how changes in probability are propagated. If we encode our probabilistic knowledge of the world by writing down $s w f f s$ and then build arguments for and against propositions using $\vdash_{A C R}$, we can identify the changes in probability of those propositions. Either way, if after building arguments and flattening we have an pair $(S t, S g)$ 


$$
\begin{aligned}
& \text { If and and then } \\
& S t=w \quad S g=\Uparrow \quad \operatorname{Pr}(w)_{\text {initial }}=p \quad \operatorname{Pr}(w)_{\text {final }}=1 \\
& S t=w \quad S g=\uparrow \quad \operatorname{Pr}(w)_{\text {initial }}=p \quad p \leq \operatorname{Pr}(w)_{\text {final }} \leq 1 \\
& S t=w \quad S g=\leftrightarrow \quad \operatorname{Pr}(w)_{\text {initial }}=p \quad \operatorname{Pr}(w)_{\text {final }}=p \\
& S t=w \quad S g=\downarrow \quad \operatorname{Pr}(w)_{\text {initial }}=p \quad p \geq \operatorname{Pr}(w)_{\text {final }}=0 \\
& S t=w \quad S g=\Downarrow \quad \operatorname{Pr}(w)_{\text {initial }}=p \quad \operatorname{Pr}(w)_{\text {final }}=0 \\
& S t=w \quad S g=\uparrow \quad \operatorname{Pr}(w)_{\text {initial }}=p \quad 0 \leq \operatorname{Pr}(w)_{\text {final }} \leq 1 \\
& \text { If and then } \\
& S t=v \rightarrow w \quad S g=++\quad \operatorname{Pr}(w \mid v, x)=1 \\
& S t=v \rightarrow w \quad S g=+-\quad \operatorname{Pr}(w \mid \neg v, x)=0 \\
& S t=v \rightarrow w \quad S g=+\quad \operatorname{Pr}(w \mid v, x) \geq \operatorname{Pr}(w \mid \neg v, x) \\
& S t=v \rightarrow w \quad S g=0 \quad \operatorname{Pr}(w \mid v, x)=\operatorname{Pr}(w \mid \neg v, x) \\
& S t=v \rightarrow w \quad S g=-\quad \operatorname{Pr}(w \mid v, x) \leq \operatorname{Pr}(w \mid \neg v, x) \\
& S t=v \rightarrow w \quad S g=-+\quad \operatorname{Pr}(w \mid \neg v, x)=1 \\
& S t=v \rightarrow w \quad S g=--\quad \operatorname{Pr}(w \mid v, x)=0 \\
& S t=v \rightarrow w \quad S g=? \quad \text { The relationship between } \operatorname{Pr}(w \mid v, x) \\
& \text { and } \operatorname{Pr}(w \mid \neg v, x) \text { is unknown. }
\end{aligned}
$$

Table 3. What a derived formula means.

where $S t$ is any $w f f$ then $S g$ indicates the change in probability of $S t$. If, on the other hand we have $(S t, S g)$ where $S t$ is an iwff $S t^{\prime} \rightarrow S t^{\prime \prime}$ then $S g$ indicates the constraint between $\operatorname{Pr}\left(S t^{\prime}\right)$ and $\operatorname{Pr}\left(S t^{\prime \prime}\right)$. The full denotation of any pair $(S t, S g)$ is given by Table 3 . Since reasoning with probability is normative in the sense that it accords to the norms of probability theory (which can be justified by the usual Dutch book argument) this makes $\mathcal{N} \mathcal{A}^{\prime \prime}$ normative.

Another advantage of $\mathcal{N} \mathcal{A}^{\prime \prime}$ is that it makes it possible to give a probabilistic analysis of different styles of argumentation. This section gives examples of three such analyses using small examples (more extensive analysis may be found in [18]). In some systems of argumentation [7], arguments are flattened by counting the number of arguments for and against a proposition and giving it the sign of the majority. Consider the following example:

$$
\begin{aligned}
& f 1: \text { embezzle_funds }: \downarrow \\
& f 2: \text { good_tutor }: \downarrow \\
& f 3: \text { good_research }: \uparrow \\
& r 1: \text { embezzle_funds } \rightarrow \text { lose_job }:+. \\
& r 2: \text { good_tutor } \rightarrow \text { lose_job }:- \\
& r 3: \text { good_research } \rightarrow \text { lose_job }:-
\end{aligned}
$$

there are three arguments affecting the proposition "lose_job"

$$
\begin{aligned}
& \Delta_{2} \vdash_{A C R}\left(l o s e_{-} j o b,(f 1, r 1), \downarrow\right) . \\
& \Delta_{2} \vdash_{A C R}\left(l o s e_{-} j o b,(f 2, r 2), \uparrow\right) . \\
& \Delta_{2} \vdash_{A C R}\left(l o s e_{-} j o b,(f 3, r 3), \downarrow\right) .
\end{aligned}
$$


Counting arguments suggests we should conclude that belief in "lose_job" decreases since there are two arguments against it and only one for it. Considering using $\mathcal{N} \mathcal{A}^{\prime \prime}$ here shows that concluding $\left\langle l o e_{-} j o b, \downarrow\right\rangle$ involves the assumption:

$$
\begin{aligned}
& \Delta \operatorname{Pr}(\text { good_tutor }) .(\operatorname{Pr}(\text { lose_job } \mid \text { good_tutor })-\operatorname{Pr}(\text { lose_job } \mid \neg \text { good_tutor })) \\
& \leq \Delta \operatorname{Pr}(\text { embezzle }) \cdot\left(\operatorname{Pr}(\text { lose_job } \mid \text { embezzle })-\operatorname{Pr}\left(l_{\text {lose_job } \mid \neg \text { embezzle })}\right)\right.
\end{aligned}
$$

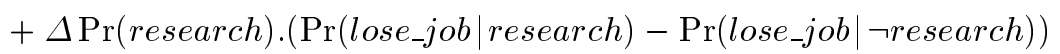

when the size of the changes in probability propagated across each implication are taken into account [19]. Another kind of flattening is that based on directness of argument [12]. With the database:

$$
\begin{aligned}
& f 1: \text { good_research }: \uparrow \\
& r 1: \text { good_research } \rightarrow \text { good_tutor }:- \\
& r 2: \text { good_tutor } \rightarrow \text { lose_job }:- \\
& r 3: \text { lose_job } \rightarrow \text { no_money }:+ \\
& r 4: \text { good_research } \rightarrow \text { job_in_industry }:+ \\
& r 5: \text { job_in_industry } \rightarrow \text { no_money }:-
\end{aligned}
$$

we get the arguments:

$$
\begin{aligned}
& \left.\Delta_{3} \vdash_{A C R} \text { (no_money, }(f 1, r 1, r 2, r 3), \uparrow\right) . \\
& \Delta_{3} \vdash_{A C R}(\text { no_money },(f 2, r 4, r 5), \downarrow) .
\end{aligned}
$$

Flattening by directness leads us to conclude $\langle$ no_money, $\downarrow\rangle$. since the argument for it is the shorter of the two. This time the probabilistic semantics of $\mathcal{N} \mathcal{A}^{\prime \prime}$ expose the assumption as being:

$$
\begin{aligned}
(\operatorname{Pr}(\text { industry } \mid \text { research })-\operatorname{Pr}(\text { industry } \mid \neg \text { research })) \\
.(\operatorname{Pr}(\text { no_money } \mid \text { industry })-\operatorname{Pr}(\text { no_money } \mid \neg \text { industry })) \\
\geq(\operatorname{Pr}(\text { good_tutor } \mid \text { research })-\operatorname{Pr}(\text { good_tutor } \mid \neg \text { research })) \\
\quad(\operatorname{Pr}(\text { lose_job } \mid \text { good_tutor })-\operatorname{Pr}(\text { lose_job } \mid \neg \text { good_tutor })) \\
\quad(\operatorname{Pr}(\text { no_money } \mid \text { lose_job })-\operatorname{Pr}(\text { no_money } \mid \neg \text { lose_job }))
\end{aligned}
$$

which is similar to that underlying counting arguments, but without placing constraints on the change in the probability of the initial facts. Finally, several authors $[11,21]$ have considered the use of rebuttal and undercutting to flatten arguments - a proposition is rebutted when it is directly argued against and is undercut when one of the steps in the argument for it is argued against. Consider the following example:

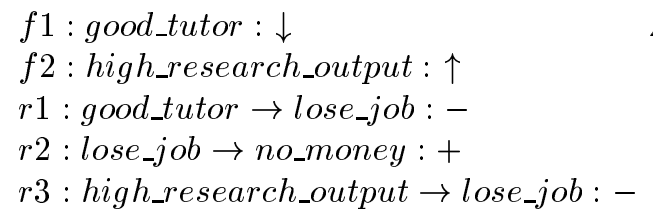


From $\Delta_{4}$ we can build the arguments:

$$
\begin{aligned}
& \Delta_{4} \vdash_{A C R}\left(l_{\text {lose_job }}(f 1, r 1), \uparrow\right) . \\
& \Delta_{4} \vdash_{A C R}(\text { no_money },(f 1, r 1, r 2), \uparrow) . \\
& \Delta_{4} \vdash_{A C R}\left(l_{\text {lose_job }}(f 2, r 3), \downarrow\right) .
\end{aligned}
$$

and find that the third argument rebuts proposition lose_job and undercuts the the proposition no_money. The usual interpretation of this is that the lose_job is more affected by the third argument than no_money, and this is exactly what one would conclude from $\mathcal{N} \mathcal{A}^{\prime \prime}$ since the changes associated with the third argument will be no smaller for $\operatorname{Pr}\left(l_{o s} e_{-}\right.$job) than for $\operatorname{Pr}\left(\right.$ no_money $\left._{\mathbf{n}}\right)$.

\section{Summary}

This paper has discussed a means of giving a probabilistic semantics to a system of argumentation. It is thus in some senses an extension of previous work on such systems of argumentation [11] as well as probabilistic systems of argumentation which use a more restricted base logic [17]. With a solid basis in probability theory, the system can be used to combine the advantages of a sound means of handling uncertainty with the expressiveness of a logical method of knowledge representation, an expressiveness that will be increased with the planned extension to a first order system which includes disjunction. Amongst other things the system is capable of defeasible reasoning and knowledge-based model construction for qualitative probabilistic networks. Furthermore, because of its qualitative nature, the system may be used when probabilistic knowledge of a domain is incomplete and the fact that it is soundly based on probability theory makes it a useful basis for a qualitative decision theory [8]. Finally, it should be noted that $\mathcal{N} \mathcal{A}^{\prime \prime}$ has similarities with Neufeld's system for default reasoning [14]. Some of these are explored in [16].

\section{References}

1. S. Benferhat, D. Dubois, and H. Prade. Argumentative inference in uncertain and inconsistent knowledge bases. In Proceedings of the 9th Conference on Uncertainty in Artificial Intelligence, 1993.

2. A. Darwiche. Argument calculus and networks. In Proceedings of the 9th Conference on Uncertainty in Artificial Intelligence, 1993.

3. A. Darwiche and M. Ginsberg. A symbolic generalisation of probability theory. In Proceedings of the 10th National Conference on Artificial Intelligence, 1992.

4. A. Darwiche and M. Goldszmidt. On the relation between kappa calculus and probabilistic reasoning. In Proceedings of the 10th Conference on Uncertainty in Artificial Intelligence, 1994.

5. M. J. Druzdzel. Probabilistic reasoning in Decision Support Systems: from computation to common sense. PhD thesis, Carnegie Mellon University, 1993.

6. P. M. Dung. On the acceptability of arguments and its fundamental role in nonmonotonic reasoning and logic programming. In Proceedings of the 13th International Conference on Artificial Intelligence, 1993. 
7. J. Fox. A unified framework for hypothetical and practical reasoning (2): lessons from clinical medicine. In Proceedings of the Conference on Formal and Applied Practical Reasoning, 1996.

8. J. Fox and S. Parsons. On using arguments for reasoning about actions and values. In Proceedings of AAAI Spring Symposium on Qualitative Preferences in Deliberation and Practical Reasoning, 1997.

9. J. Fox, S. Parsons, P. Krause, and M Elvang-Gørannson. A generic framework for uncertain reasoning. In Proceedings of the IMACS III International Workshop on Qualitative Reasoning and Decision Technologies, 1993.

10. M. Goldszmidt and J. Pearl. Qualitative probabilities for default reasoning, belief revision and causal modelling. Artificial Intelligence, 84:57-112, 1996.

11. P. Krause, S. Ambler, M. Elvang-Gøransson, and J. Fox. A logic of argumentation for reasoning under uncertainty. Computational Intelligence, 11:113-131, 1995.

12. R. Loui. Defeat among arguments: a system of defeasible inference. Computational Intelligence, 3:100-106, 1987.

13. N. F. Michelena. Monotonic influence diagrams: application to optimal and robust design. PhD thesis, University of California at Berkeley, 1991.

14. E. Neufeld. A probabilistic commonsense reasoner. International Journal of Intelligent Systems, 5:565-594, 1990.

15. S. Parsons. Refining reasoning in qualitative probabilistic networks. In Proceedings of the 11th Conference on Uncertainty in Artificial Intelligence, 1995.

16. S. Parsons. Comparing normative argumentation with other qualitative probabilistic systems. In Proceedings of the Conference on Information Processing and the Management of Uncertainty, 1996.

17. S. Parsons. Defining normative systems for qualitative argumentation. In Proceedings of the Conference on Formal and Applied Practical Reasoning, 1996.

18. S. Parsons. Normative argumentation and qualitative probability. Technical report, Department of Electronic Engineering, Queen Mary and Westfield College, 1996.

19. S. Parsons. Qualitative approaches to reasoning under uncertainty. MIT Press, (to appear), Cambridge, MA, 1997.

20. J. Pearl. Probabilistic reasoning in intelligent systems; networks of plausible inference. Morgan Kaufmann, San Mateo, CA., 1988.

21. J. L. Pollock. Justification and defeat. Artificial Intelligence, 67:377-407, 1994.

22. M. P. Wellman. Formulation of tradeoffs in planning under uncertainty. Pitman, London, 1990.

23. N. Wilson. An order of magnitude calculus. In Proceedings of the 11th Conference on Uncertainty in Artificial Intelligence, 1995. 